\title{
Bullosis Diabeticorum: A Diabetic Bullosis, Commonly Unknown
}

\author{
Kyo Joon Kang, Sung-No Jung, Bommie Florence Seo \\ Department of Plastic and Reconstructive Surgery, Uijeongbu St. Mary's Hospital, College of Medicine, The Catholic University of Korea, Uijeongbu, Korea
}

\begin{abstract}
Bullosis diabeticorum (BD) is a distinct, spontaneous, noninflammatory, and blistering lesion that develops predominantly in the acral skin of patients with diabetes mellitus (DM). BD is a rare disease that develops in patients with longterm DM and not well known to physicians and often fails to be diagnosed. The lesions are known to spontaneously heal in most patients in 2 to 6 weeks. Wound care is required to prevent secondary infection of the blisters. However, if infection develops and progresses to necrosis, surgical intervention including debridement and reconstruction may be necessary. We report a case of BD in a patient with relatively well controlled $\mathrm{DM}$, whose wounds progressed to necrosis and then spontaneously healed with dressings.
\end{abstract}

Keywords: Bullosis diabeticorum, Infection, Necrosis

\section{Introduction}

Bullosis diabeticorum (BD) is a relatively rare, spontaneous, noninflammatory, and blistering entity that develops predominantly in the acral skin of patients with diabetes mellitus (DM) [1]. BD develops mainly in long-standing diabetic patients. However, the exact pathogenesis of BD is yet to be elucidated, but is thought to be multifactorial. It has been reported in the United States that BD develops in approximately $0.5 \%$ of patients with DM and that the incidence in men is twice of that in women $[2,3]$. BD is known to spontaneously heal in most patients within 2 to 6 weeks. However, if infection develops and progresses to necrosis, surgical treatment may be necessary [4].

We report a case of $\mathrm{BD}$ that developed in the lower extremities that partially progressed into a necrotic wound, then healed spontaneously. This brief depiction aims to emphasize that $\mathrm{BD}$ may be a potential diagnosis in such blistering wounds, and to aid the clinician in choosing adequate management.

\section{Case report}

A 68-year-old male presented with multiple spontaneous bullae that had developed several days ago on both his feet. He had been diagnosed 30 years ago with type II DM for which he was being treated with medication. He had received stent treatment for coronary artery disease and had chronic kidney disease. His daily blood glucose levels were between $80 \mathrm{mg} / \mathrm{dL}$ to $180 \mathrm{mg} / \mathrm{dL}$. Hemoglobin A1c at presentation was 7.5\%.

Upon physical examination the patient had warm feet with good palpation of both dorsalis pedis arteries. He had undergone percutaneous transluminal angioplasty of both lower legs at another hospital a few years ago. There was no swelling of the lower

\author{
Case Report \\ Received: May 9, 2018 \\ Revised: August 31, 2018 \\ Accepted: September 1, 2018 \\ Corresponding author: \\ Bommie Florence Seo, M.D. \\ Department of Plastic and Reconstructive \\ Surgery, Uijeongbu St. Mary's Hospital, College \\ of Medicine, The Catholic University of Korea, \\ 271 Chenbo-ro, Uijeongbu 11765, Korea \\ Tel: +82-31-820-3935 \\ Fax: +82-31-874-0301 \\ E-mail: bommiefseo@gmail.com
}

This is an Open Access article distributed under the terms of the Creative Commons Attribution Non-Commercial License (http://creativecommons.org/licenses/by-nc/4.0/) which permits unrestricted non-commercial use, distribution, and reproduction in any medium, provided the original work is properly cited.

C 2018 Korean Wound Management Society 
extremities, and no discoloration. Several days before visiting the hospital, multiple spontaneous bullae had developed without any friction, contusion, or strangulation history on both feet. The bullae were located over the navicular tubercle area of both feet, the medial aspect of the left heel, the medial aspect of the first left metatarsal, the lateral aspect of the left heel and a curvilinear area anterior to the lateral malleolus of the left foot. The diameters of the bullae ranged from $1.5 \mathrm{~cm}$ to $4 \mathrm{~cm}$. The bullae had a non erythematous base, were filled with serous fluid and there were no surrounding infection signs. There was no history of dermatologic disorder, no acute edema and no change in medication history. He had not changed footwear, wore loose, well aerated sneakers, and loose socks. He reported that he had experienced spontaneous development and subsequent healing of bullae on his shoulders, abdomen, knee, and upper extremities before, beginning around 3 years ago. The lesions occurred at intervals of 3 to 4 months and spontaneously healed 1 to 2 weeks after the lesion occurred. The patient had visited the hospital this time because this was the first time these lesions had occurred on his feet, and he had been warned about the dire consequences of untreated diabetic foot patients and was anxious. However, he refused admission or extensive laboratory workup, and agreed to dressings in the outpatient environment.
Bullectomy and foam dressing were performed, and the wounds were followed up at weekly intervals. The patient was compliant and was educated to dress his feet every other day at home in an aseptic manner. The wounds demarcated over the span of 3 weeks, and slow marginal epithelialization progressed.

At three weeks after bullectomy, infection signs including swelling, erythema and tenderness developed on the left foot (Fig. 1). The medial wound of the left foot had suddenly progressed into an ulcer centrally covered with a necrotic yellow soft eschar. Wound cultures and a punch biopsy of $5 \mathrm{~mm}$ diameter of the central portion of the left medial wound were obtained. We strongly advised admission and close observation, but the patient refused, so intravenous antibiotics (Flomoxef sodium, Isepamicin sulfate) and oral antibiotics (Cefaclor) were administered for one week. Cultures revealed growth of Staphylococcus aureus and Pseudomonas aeruginosa. Susceptibility results revealed that the Pseudomonas aeruginosa was susceptible to amikacin and gentamicin and the Staphylococcus aureus was susceptible to oxacillin. By the following week, infection signs had almost diminished. By 6 weeks after the initial visit, the bullae had proceeded to a fully demarcated necrotic wound (Fig. 2).

At 8 weeks after the initial visit, two bullae developed on the
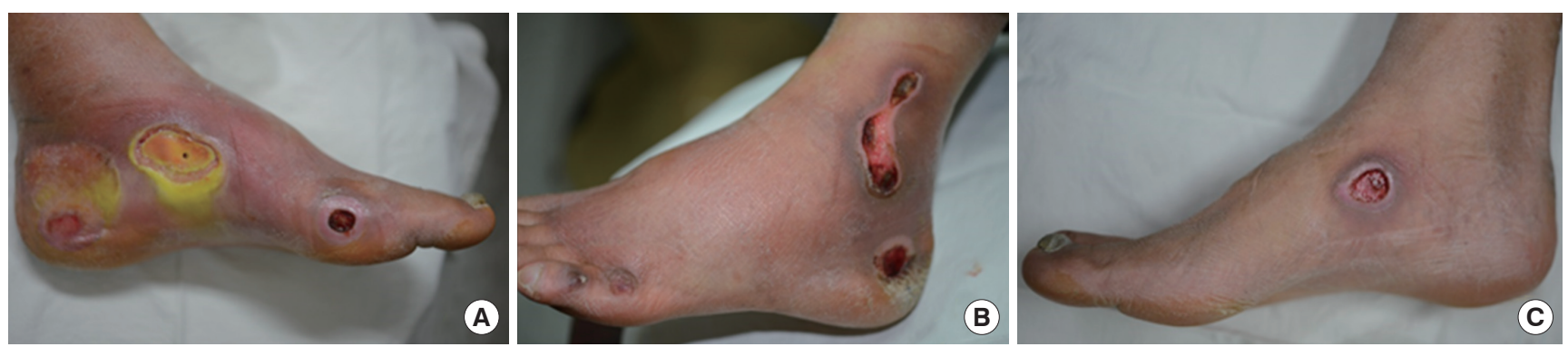

Fig. 1. Infectious change of the left foot. Two months after bullectomy, erythema and swelling were observed in the heel area (A) Medial aspect of left foot. (B) Lateral aspect of left foot. (C) Medial aspect of right foot.
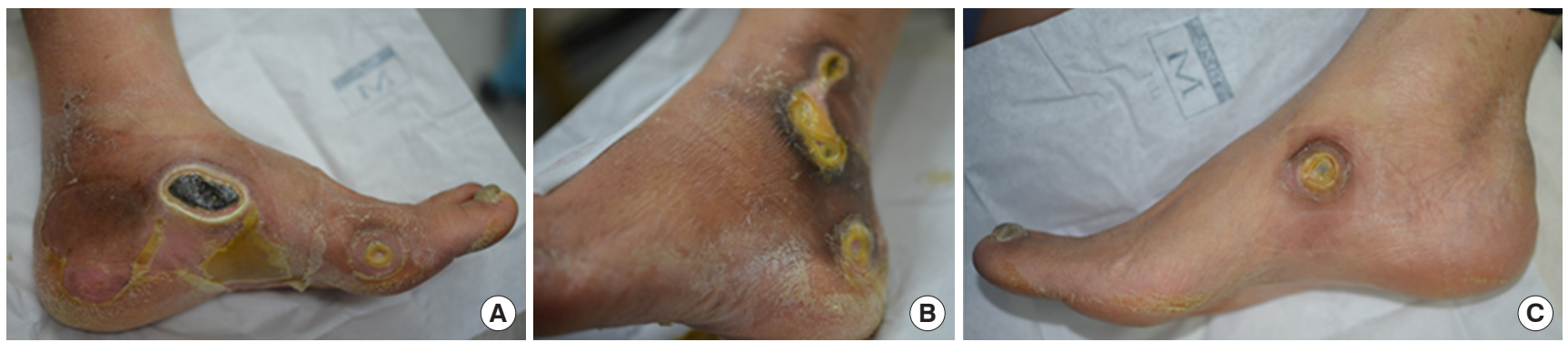

Fig. 2. Progression of the wounds. Demarcation is seen. (A) Medial aspect of left foot. (B) Lateral aspect of left foot. (C) Medial aspect of right foot. 
patient's upper right arm and spontaneously healed after one week with simple dressings.

Hematoxylin and eosin staining of the perilesional skin biopsy obtained during inflammatory progression of the necrotic area showed necrotic fibrous tissue with several eosinophils and inflammatory cells (Fig. 3). No further immunohistochemistry studies were done.

After 16 weeks of simple weekly dressings using normal saline, Mupirocin ointment and foam, all wounds underwent marginal epithelialization and contracture, with no further incidents of infection (Fig. 4).

The patient agreed to share his experience via signed informed consent.

\section{Discussion}

Bullosus diabeticorum is one of many cutaneous manifestations of DM. It is a blister lesion that develops suddenly in pa-

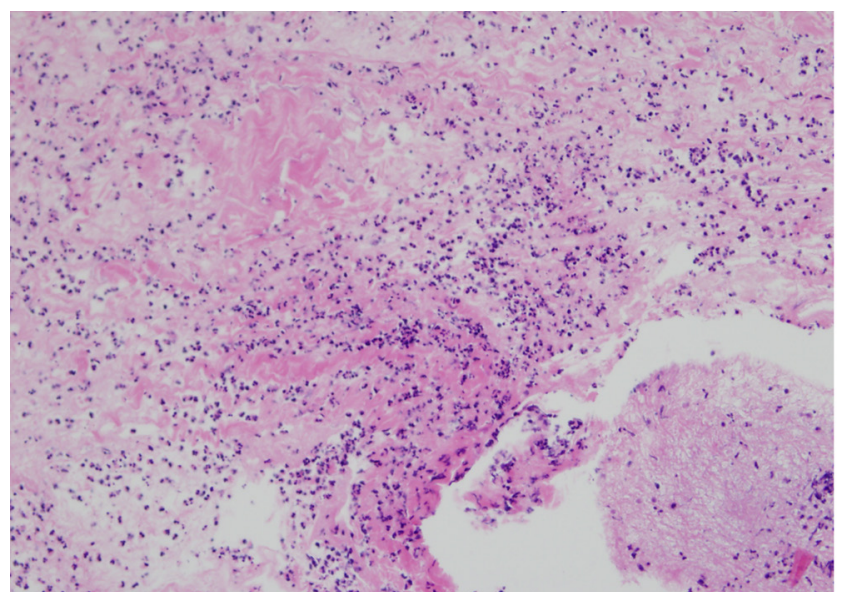

Fig. 3. Microscopic view of the specimen. Necrotic fibrous tissue with several eosinophils and inflammatory cells are seen (Hematoxylin and eosin stain, $\times 200$ ). tients, usually with long-standing DM, with no apparent cause or trauma history. Blisters are mostly painless, asymmetrical, and non-pruritic. Occasionally, they will grow with irregular margins and are flaccid, filled with serous fluid, similar to burn lesions. The lesions are most commonly found on the acral and distal skin, but rarely do develop in non-acral sites, such as the trunk [3]. The pathogenesis of BD has not been clearly elucidated, and is suspected to be multifactorial. There have been no definitive studies correlating the degree of diabetic control with the presence of these lesions. Wilson et al. reviewed a single patient during an 11 year period, comparing blood glucose levels during occurence of $\mathrm{BD}$ with levels when he had no BD. They found that bullae formation was more likely to occur when blood glucose levels where elevated $[4,5]$. However, studies of a larger group of patients are required before any conclusions can be made. The patient in our case had relatively well controlled blood glucose, although a long term comparison was not performed. Some have even hypothesized that $\mathrm{BD}$ may be found in the prediabetic state, even before diabetes is diagnosed [6]. A long period of microvascular disease may cause thickening of the capillary basement membrane, that may lead to tissue hypoxia and blister formation [7]. There have been reports of BD in patients with nephropathy, microangiopathy, disorders in the regulation of carbohydrates, calcium, and magnesium [8]. Ultraviolet-ray exposure has been suggested as a cause, and it has also been postulated that blister formation may occur due to elevated porphyrin levels in patients with end-stage renal failure $[3,8]$.

Diagnosis of $\mathrm{BD}$ is a diagnosis of exclusion. The history reveals a nontraumatic, spontaneously occurring blister, one that may repeatedly occur in different sites of the body. This history will help exclude the commonly found frictional bullae that easily progresses to ulceration. There are no specific laboratory tests that aid diagnosis. Histopatholic findings are not very
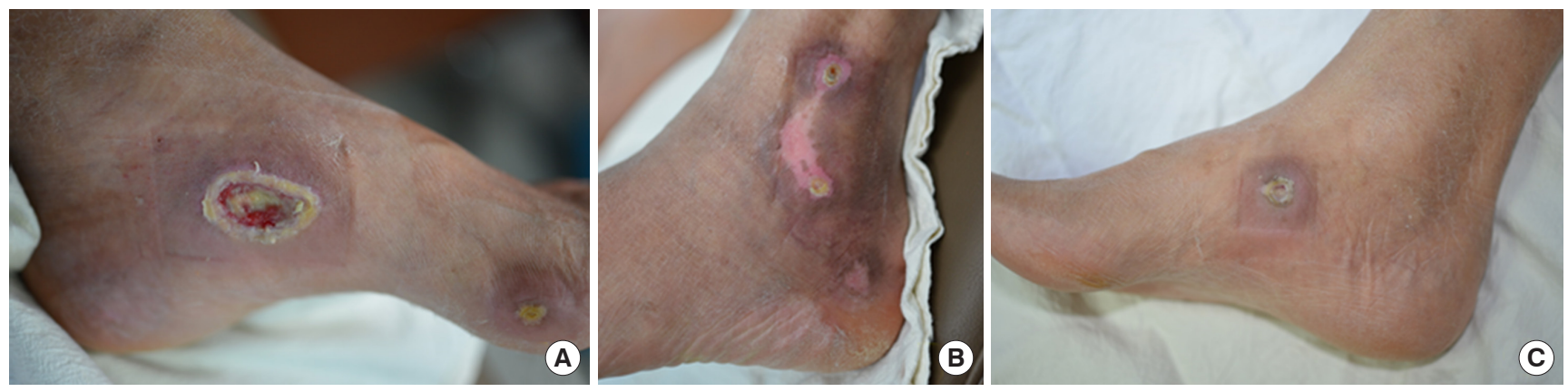

Fig. 4. Both feet after 16 weeks. Both feet show epithelialization of all wounds without any infection signs. (A) Medial aspect of left foot. (B) Lateral aspect of left foot. (C) Medial aspect of right foot. 
specific, usually revealing an intraepidermal or subepidermal blister with a scanty to moderate inflammatory cell proliferate. Immunofluorescence staining tests typically appears negative for IgM, IgG, IgA, and complement C3. Such clinical features should elicit suspicion of the diagnosis of $\mathrm{BD}$, which should be then distinguished from other vesicobullous disorders such as bullous pemphigoid, epidermolysis bullosa, epidermolysis bullosa acquisita, porphyria cutanea tarda, pseudoporphyria, and drug-induced bullous disorders via histology and immunofluorescence $[3,9]$. The absence of immunologic abnormalities is not universal though, there has been on case that found IgG deposition in the basement membrane of the superficial capillaries, and another that reported positive findings of nonspecific capillary-associated IgM and C3 [10].

Because $\mathrm{BD}$ is mainly self-limiting, there is no need for special treatment. Blisters typically spontaneously heal within 2 to 6 weeks. However, wound infection can lead to necrosis [11]. There has been a report of $\mathrm{BD}$ associated with osteomyelitis [11]. Infection of the wounds require prompt intervention with intravenous antibiotics and intensive wound care. $\mathrm{Ne}-$ crotic lesions may necessitate serial debridement and coverage using skin grafts, biological dressings, or healing with secondary intention. Even after successful treatment of acute wounds, the patient must also be warned about recurrence. Some researchers have strived to find a method to prevent recurrence. Chen et al. have reported that autologous bone marrow mesenchymal cell transplantation therapy in a diabetic patient that suffered from recurrent $\mathrm{BD}$ on five occasions was effective, the patient was recurrence-free for the 10 years following transplantation [7]. Although the results are promising, further investigation is needed to be conclusive.

In conclusion, bullosis diabeticorum is a rare cutaneous manifestation of diabetes mellitus, that is not well known to physicians and often fails to be diagnosed. While the majority of lesions is self-limiting, meticulous wound care should be performed to prevent secondary infection. The physician should be aware of this entity, and have a high index of suspicion when encountering such bullous presentations.

\section{Conflict of interest}

No potential conflict of interest relevant to this article was reported.

\section{References}

1. Kramer DW. Early or warning signs of impending gangrene in diabetes. Med J Rec 1930;132:338-42.

2. Cantwell AR Jr, Martz W. Idiopathic bullae in diabetics. BulIosis diabeticorum. Arch Dermatol 1967;96:42-4.

3. Medscape. 2015 Bullous disease of diabetes [Internet]. Medscape;updated 2015 [cited 27 Jul 2017]. Available from: http:// emedicine.medscape.com/article/1062235

4. Wilson TC, Snyder RJ, Southerland CC. Bullosis diabeticorum: is there a correlation between hyperglycemia and this symptomatology? Wounds 2012;24:350-5.

5. Lipsky BA, Baker PD, Ahroni JH. Diabetic bullae: 12 cases of a purportedly rare cutaneous disorder. Int J Dermatol 2000; 39:196-200.

6. Lopez PR, Leicht S, Sigmon JR, et al. Bullosis diabeticorum associated with a prediabetic state. South Med J 2009;102: 643-4.

7. Chen Y, Ma Y, Li N, et al. Efficacy and long-term longitudinal follow-up of bone marrow mesenchymal cell transplantation therapy in a diabetic patient with recurrent lower limb bullosis diabeticorum. Stem Cell Res Ther 2018;9:99.

8. Cantwell AR, Martz A. Idiopathic bullae in diabetics. Bullosis diabeticorum. Arch Dermatol 1967;96:42-4.

9. Sonani H, Abdul SS, Garla VV, et al. Bullosis diabeticorum: a rare presentation with immunoglobulin $\mathrm{G}$ (IgG) deposition related vasculopathy. Case report and focused review. Am J Case Rep 2018;15:52-6.

10. Larsen $\mathrm{K}$, Jensen T, Karlsmark T, et al. Incidence of bullosis diabeticorum - a controversial cause of chronic foot ulceration. Int Wound J 2008;5:591-96.

11. Tunuguntla A, Patel KN, Peiris AN, et al. Bullosis diabeticorum associated with osteomyelitis. Tenn Med 2004;97:5034. 